\title{
Avaliação da função hepática em cães submetidos a anestesia pela associação Zolazepam/Tiletamina
}

\author{
Hepatic function evolution in dogs anesthetized \\ with Zolazepam/Tiletamine
}

\author{
Ewaldo de Mattos Junior ${ }^{*}$; Claudia Russo²; Gustavo José Von Glehn Santos; \\ Mara Regina Stripp Balarin ${ }^{4}$; João Paulo Elsen Saut ${ }^{5}$
}

\begin{abstract}
Resumo
Foram avaliados os efeitos da associação zolazepam/tiletamina sobre o fígado por meio da alterações nas concentrações séricas de enzimas hepáticas. Foram utilizados dez cães clinicamente hígidos, distribuídos em dois grupos de cinco animais designados como grupo I (GI) e grupo II (GII). Os animais de GI foram submetidos a $6,6 \mathrm{mg} / \mathrm{kg}$ de zolazepam/tiletamina, por via intramuscular em dose única. No GII, aplicouse $6,6 \mathrm{mg} / \mathrm{kg}$ de zopazepam/tiletamina pela mesma via; decorridos 40 e 80 minutos, os animais receberam outras duas aplicações de $3,3 \mathrm{mg} / \mathrm{kg}$ por via intravenosa. As provas de função hepática foram realizadas por meio das dosagens de alanina amino transferase (ALT), aspartato amino transferase (AST), fosfatase alcalina (FA) e gama glutamil transferase (GGT). As amostras foram obtidas nos momentos M0 até M4, sendo: M0 anterior a aplicação da medicação; M1 até M3 com intervalos de 20 minutos após M1; e M4 24 horas após M1. Os dados foram submetidos ao teste de normalidade de Kolmogorov-Smirnov, e o teste de Tukey foi utilizado na comparação das médias das variáveis. O nível de significância estabelecido foi de 5\%. Os valores médios de todas as enzimas avaliadas permaneceram dentro da normalidade em ambos os grupos, não apresentando variações dignas de nota nem estatisticamente significativas entre momentos bem como entre grupos. Diante dos resultados obtidos nesse experimento, conclui-se que: a associação anestésica zolazepam/tiletamina na dose de $6,6 \mathrm{mg} / \mathrm{kg}$, seguida de duas novas aplicações de $3,3 \mathrm{mg} / \mathrm{kg}$ elevam as enzimas hepáticas avaliadas em cães, sem ultrapassar os valores fisiológicos; uma dose de $6,6 \mathrm{mg} / \mathrm{kg}$ de zolazepam/tiletamina por via intramuscular promove valores menores das enzimas avaliadas em relação a três doses.
\end{abstract}

Palavras-chave: Anestesia dissociativa, ezimas hepáticas, caninos

\begin{abstract}
The hepatic effects of the anesthetic association zolazepam/tiletamine were investigated in dogs by analyzing the serum concentration of hepatic enzymes. Ten healthy dogs were divided into two groups of five, group I (GI) and group II (GII). The animals of GI received a single dose of $6,6 \mathrm{mg} / \mathrm{kg}$ of zolazepam/tiletamine, by intramuscular (IM) injection. GII dogs received $6,6 \mathrm{mg} / \mathrm{kg}$ of zolazepam/ tiletamine by the IM route; after a period of 50 - 80 minutes the animals received two additional doses
\end{abstract}

1 Médico Veterinário, MSc, Aluno de pós-graduação do departamento de cirurgia da Faculdade de Medicina Veterinária e Zootecnia, Universidade de São Paulo, São Paulo, SP. E-mail: ewaldomattos@hotmail.com

2 Médico Veterinário, MSc, Departamento de Cirurgia, Curso de Medicina Veterinária, Universidade Luterana do Brasil, Ji-Paraná, RO..

3 Médico Veterinário, Dr, Departamento de Cirurgia Experimental, Faculdade de Medicina, Universidade Regional de Gurupi, Gurupi - TO.

4 Médica Veterinária, Dr, Departamento de Patologia Clínica, Curso de Medicina Veterinária, Universidade Estadual de Londrina, Londrina - PR.

5 Médico Veterinário, Dr, Departamento de Clinica Médica, Curso de Medicina Veterinária, Universidade Federal de Uberlândia, Uberlândia, MG.

* Autor para correspondência 
$(3,3 \mathrm{mg} / \mathrm{kg})$ by intravenous administration[SAH1]. The hepatic function were analyzed by monitoring the serum concentrations of alanine aminotransferase (ALT), aspartate aminotransferase (AST), alkaline phosphatase (ALP), and gamma-glutamyl-transferase (GGT). Four blood samples were collected in different moments during the analyses: M0, before the first application of the drug; and M1 to M4. M1 through M3 was collected with intervals of 20 minutes before M0, while M4 was obtained 24 hours after M1. The normality of the obtained results was analyzed by Kolmogorov-Smirnov Test; while the Tukey's test compared the means, using a level of significance of 5\% for both statistical analyses. The mean values of all enzymes evaluated were within normal limits for both experimental groups, without any significant statistical alteration being observed between and within these groups. These results demonstrated that the association of zolazepam/tiletamine at the dosage of $6.6 \mathrm{mg} / \mathrm{kg}$, followed by two applications additional of $3.3 \mathrm{mg} / \mathrm{kg}$ resulted in elevation of the evaluated hepatic enzymes without exceeding the physiologic values. Additionally, a single application of $6.6 \mathrm{mg} / \mathrm{kg}$ of zolazepam/ tiletamine by the intramuscular route resulted in lower values when compared to three applications.

Key words: Dissociative anesthesia, hepatic enzymes, canine

\section{Introdução}

Várias associações anestésicas fundamentadas em fármacos dissociativos têm sido amplamente utilizadas experimentalmente ou mesmo na rotina clínico-cirúrgica veterinária, sendo estas associações consideradas, sob o ponto de vista prático, como seguras e eficientes (DUPRAS et al. 2001; HEAVNER, 1996). Desta forma, as associações anestésicas baseadas em tais fármacos consagraramse na rotina anestesiológica veterinária devido à facilidade com as quais são comercializadas, associadas ao fato da praticidade de seu uso, uma vez que, em dose única por via intramuscular, o próprio cirurgião realiza o procedimento anestésico (MASSONE, 2003).

Derivadas das fenciclidinas, a cetamina e a tiletamina são os dois agentes utilizados na atualidade e produzem uma anestesia do tipo cataleptóide, com perda da movimentação voluntária, rigidez e extensão parcial dos membros, sendo o estado dissociativo aparentemente causado pela interrupção dos impulsos no sistema nervoso central e depressão diferenciada em várias áreas do encéfalo (MELLO; CORDEIRO, 2001). Valadão (2002) descreve cinco mecanismos de ação dos agentes dissociativos, sendo eles: 1) antagonismo não competitivo dos receptores $\mathrm{N}$-metil-D-aspartato (NMDA), envolvidos com a condução dos impulsos sensoriais espinhal, talâmico, límbico, subcortical (gânglio da base) e cortical. 2) ação Gabaérgica direta. 3) bloqueio de recaptação de catecolaminas. 4) agonismo dos receptores opióides Sigma na medula espinhal. 5) antagonismo dos receptores muscarínicos do SNC.

A tiletamina possui potência maior do que a cetamina, podendo ser utilizada em diversos procedimentos como medicação préanestésica, sedação, imobilização e anestesia para procedimentos pouco invasivos em cães, gatos, ruminantes, eqüinos e animais silvestres (SAVVAS et al., 2005). É comercializada na associação de 1:1 com o zolazepam, potente benzodiazepínico, o qual promove grande ação relaxante muscular (SANTOS et al., 2004).

Após a aplicação intramuscular, a associação zolazepam/tiletamina apresenta absorção e distribuição rápida, com duração de ação em torno de 45 minutos, seguida de uma fase de eliminação longa com duração de horas, fato observado por Mello e Cordeiro (2001). Semelhante ao que acontece com a cetamina, a tiletamina não se liga intensamente às proteínas plasmáticas, sendo a ligação da primeira oscilando entre 27 e 47\% (DUVAL NETO, 2004).

As fenciclidinas são biotransformadas por meio do sistema microssomal hepático, sendo a maior parte do processo envolvendo a $N$-desmetilação. Em se tratando da cetamina, após tal processo, gera-se a norcetamina (metabólito I), que sofre hidroxilação, passando a hidroxinorcetamina (DUVAL NETO, 2004). Tal produto é conjugado em derivados 
glicurônicos solúveis em água e excretados na urina. Ainda não foi esclarecido a atividade farmacológica desse último metabólito em relação ao fármaco principal,, no entanto, sabe-se que o metabólito I possui ação anestésica de 20 - 30\% comparado a cetamina (REVES; GLASS; LUBARSKY, 2000). Por isso talvez, a recuperação apresenta-se lenta e com presença de efeitos colaterais de deambulação, dificuldade de permanecer em estação e movimentos de pedalagem com a utilização de cetamina ou tiletamina em cães (SANTOS et al., 2004) e em humanos com alucinações e incordenação se estendendo por até dias após a utilização de cetamina (REVES; GLASS; LUBARSKY, 2000).

Apesar de ser amplamente utilizado na rotina anestesiológica veterinária são escassos os trabalhos sobre a avaliação da associação zolazepam/ tiletamina e as alterações que os agentes em questão podem promover sobre as enzimas hepáticas alanina amino transferase (ALT), aspartato amino transferase (AST), fosfatase alcalina (FA) e gama glutamil transferase (GGT). Essas por sua vez são utilizadas para avaliar os danos hepáticos causados por fármacos ou outras enfermidades, caracterizados por respostas agudas expressas pela ALT e AST ou crônicas observando aumentos de FA e GGT (MEYER; COLES; RICH, 1995; TENNANT, 1997). Nesse sentido, o presente trabalho tem por objetivo identificar e descrever as possíveis alterações nas enzimas hepáticas e conseqüentemente, danos ao fígado em cães submetidos a essa associação.

\section{Materiais e Métodos}

Foram utilizados dez cães, clinicamente hígidos, com peso variando entre $13 \pm 1,9 \mathrm{~kg}$, machos ou fêmeas, não utilizando fêmeas gestantes ou em estro, provenientes do biotério da Faculdade de Medicina Veterinária da Faculdades Integrado. Todos os animais foram submetidos à avaliação hematológica completa e perfil bioquímico renal e hepático a fim de evitar qualquer enfermidade que pudesse alterar os dados. Os animais foram distribuídos em dois grupos de cinco animais, designados como grupo I (GI) e grupo II (GII). Os animais de GI foram submetidos a $6,6 \mathrm{mg} / \mathrm{kg}$ de zolazepam/tiletamina ${ }^{5 a}$ por via intramuscular e 40 minutos após a primeira, receberam nova aplicação de $3,3 \mathrm{mg} / \mathrm{kg}$ por via intravenosa e uma terceira aplicação na mesma dose e via 40 minutos após a segunda. Os animais de GII receberam $6,6 \mathrm{mg} / \mathrm{kg}$ de zolazepam/tiletamina, por via intramuscular em dose única. As provas de função hepática foram realizadas através das dosagens de alanina amino transferase (ALT), aspartato amino transferase (AST), fosfatase alcalina (FA) e gama glutamil transferase (GGT). As amostras foram obtidas nos momentos M0 até M4, sendo: M0 imediatamente anterior a aplicação do fármaco; M1 quinze minutos após M0; M2 e M3 com intervalos de 20 minutos após M1 e M4 24 horas após M1. Os dados foram submetidos ao teste de normalidade de Kolmogorov-Smirnov, e o teste de Tukey foi utilizado na comparação das médias das variáveis. $\mathrm{O}$ nível de significância estabelecido foi de $5 \%$.

\section{Resultados}

Os dados referentes aos valores alanina amino transferase (ALT) estão contidos na tabela 1.

Não houve diferença comparativamente entre os momentos avaliados, sendo que estes permaneceram nos limites fisiológicos em todos os momentos e grupos, no entanto, percebe-se elevação do referido parâmetro até M3, obtendo queda gradativa até M4 (Figura 1). Apesar de não haver diferença estatística entre os grupos, observou-se valores superiores em GI em relação à GII.

$\mathrm{Na}$ avaliação estatística na atividade da enzima aspartato amino transferase (AST), não foram verificados diferençasignificativa comparativamente entre momentos e grupos $(\mathrm{p}>0,05)$ (Tabela 2, Figura 2).

\footnotetext{
5a Telazol, Fort-Dodge, São Paulo, SP.
} 
Tabela 1. Variação dos valores médios ( $\bar{x}$ ), coeficientes de variação (CV) e desvios-padrão (s) da ALT (U/L) em cães anestesiados com a associação zolazepam/tiletamina em diferentes momentos.

\begin{tabular}{ccccccc}
\hline & & M0 & M1 & M2 & M3 & M4 \\
\hline \multirow{4}{*}{ GI } & $\bar{x}$ & 13,51 & 19,94 & 21,90 & 25,05 & 17,45 \\
& $\mathbf{C V}$ & 4,37 & 7,16 & 8,22 & 8,15 & 6,58 \\
& $\mathbf{S}$ & 9,77 & 16,02 & 18,28 & 18,22 & 16,77 \\
\multirow{3}{*}{ GII } & $\bar{x}$ & 8,38 & 13,38 & 15,61 & 17,95 & 12,65 \\
& $\mathbf{C V}$ & 1,19 & 2,41 & 2,56 & 3,52 & 1,63 \\
& $\mathbf{S}$ & 2,76 & 2,38 & 4,81 & 8,38 & 4,16 \\
\hline
\end{tabular}

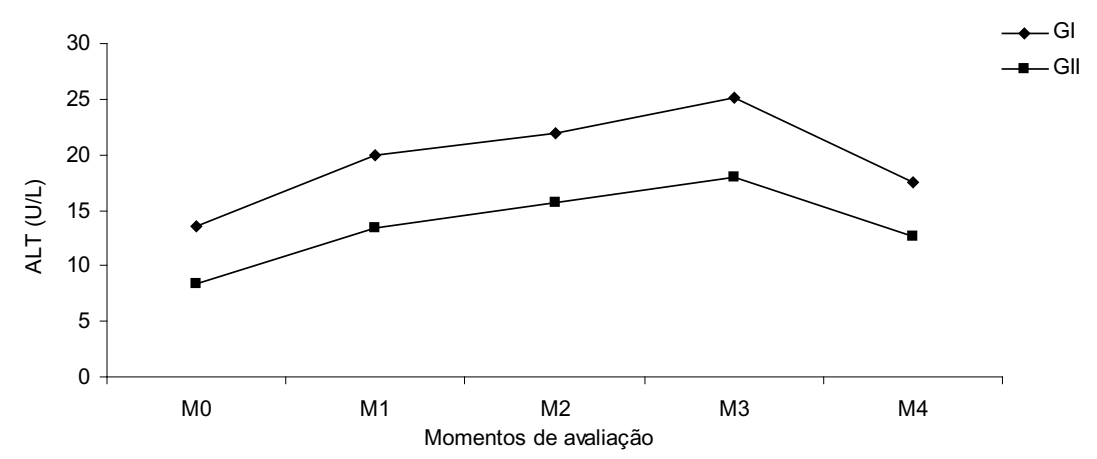

Figura 1. Variação dos valores médios da ALT (U/L) em cães anestesiados com a associação zolazepam/tiletamina em diferentes momentos.

Tabela 2. Variação dos valores médios ( $\bar{x}$ ), coeficientes de variação (CV) e desvios-padrão (s) da AST (U/L) em cães anestesiados com a associação zolazepam/tiletamina em diferentes momentos.

\begin{tabular}{ccccccc}
\hline & & M0 & M1 & M2 & M3 & M4 \\
\hline \multirow{4}{*}{ GI } & $\bar{x}$ & 7,72 & 8,63 & 10,22 & 11,76 & 9,66 \\
& $\mathbf{C V}$ & 1,87 & 1,51 & 1,95 & 2,33 & 2,03 \\
& $\mathbf{s}$ & 2,91 & 2,04 & 2,82 & 4,29 & 1,91 \\
& $\bar{x}$ & 7,43 & 8,60 & 10,25 & 12,60 & 9,48 \\
\multirow{3}{*}{ GII } & $\mathbf{C V}$ & 0,54 & 0,75 & 0,99 & 2,01 & 1,09 \\
& $\mathbf{s}$ & 1,13 & 0,77 & 1,02 & 3,13 & 1,13 \\
\hline
\end{tabular}

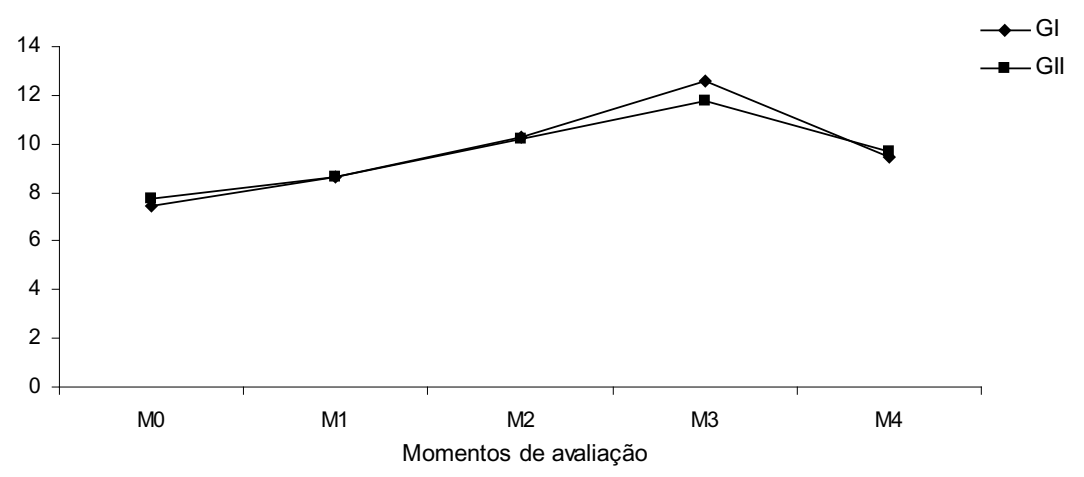

Figura 2. Variação dos valores médios da AST (U/L) em cães anestesiados com a associação zolazepam/tiletamina em diferentes momentos. 
Com relação à fosfatase alcalina (FA), não verificou-se diferença na avaliação entre momentos e grupos $(\mathrm{p}>0,05)$ (Tabela 3). Vale ressaltar que em ambos os grupos houve incremento dos valores até M3, apresentando redução até M4, sendo este mais expressivo em GI (Figura 3).
Os valores de gama glutamil transferase (GGT) não apresentaram diferença significante entre momentos ou grupos (Tabela 4). Os resultados de ambos os grupos apresentaram comportamento semelhante, apesar dos valores de GI serem superiores (Figura 4).

Tabela 3. Variação dos valores médios ( $\bar{x}$ ), coeficientes de variação (CV) e desvios-padrão (s) da FA (U/L) em cães anestesiados com a associação zolazepam/tiletamina em diferentes momentos.

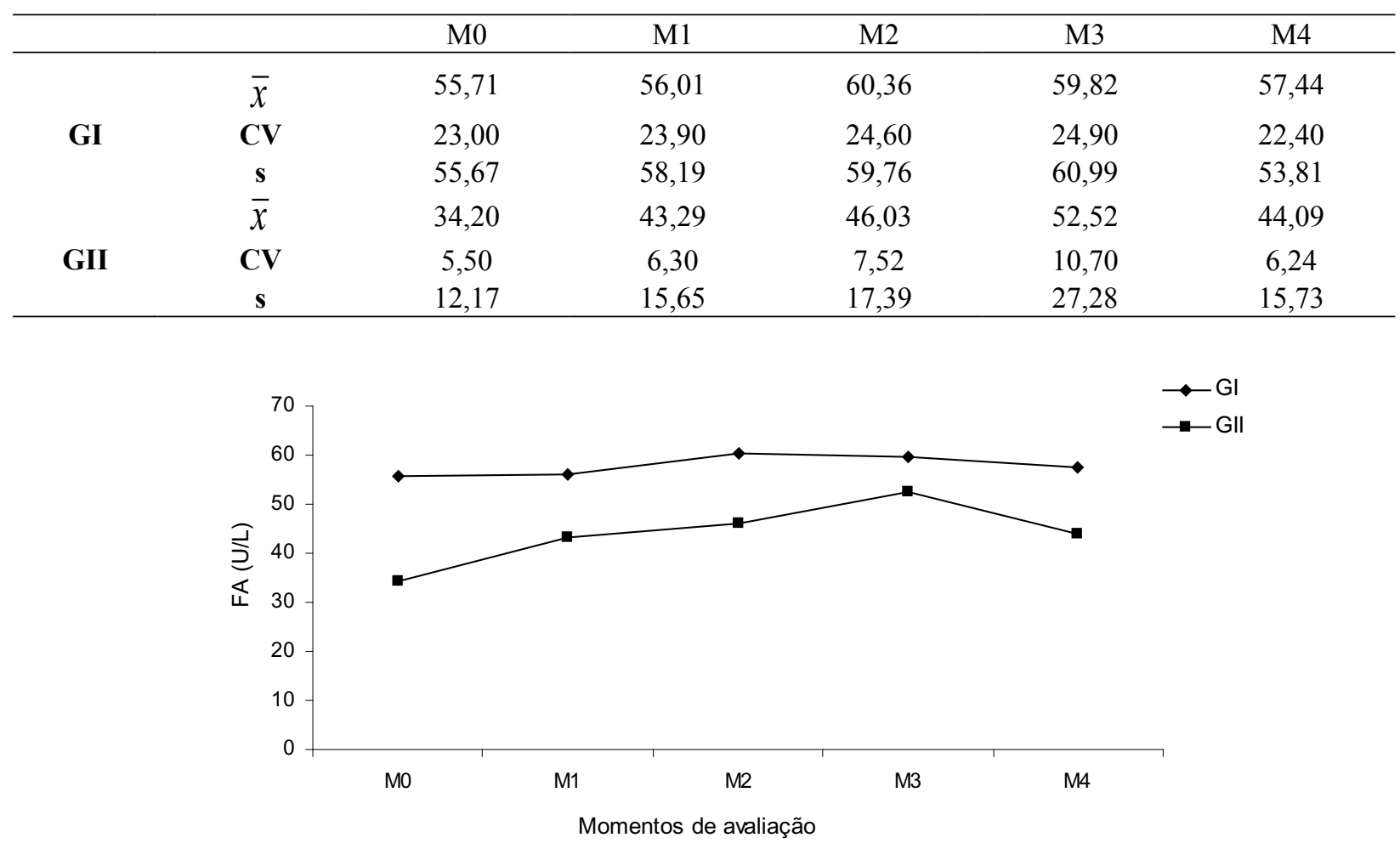

Figura 3. Variação dos valores médios da FA (U/L) em cães anestesiados com a associação zolazepam/tiletamina em diferentes momentos

Tabela 4. Variação dos valores médios ( $\bar{x}$ ), coeficientes de variação (CV) e desvios-padrão (s) da GGT (U/L) em cães anestesiados com a associação zolazepam/tiletamina em diferentes momentos.

\begin{tabular}{ccccccc}
\hline & & M0 & M1 & M2 & M3 & M4 \\
\hline \multirow{3}{*}{ GI } & $\bar{x}$ & 2,65 & 2,71 & 3,47 & 3,76 & 2,97 \\
& $\mathbf{C V}$ & 0,54 & 0,51 & 0,36 & 0,32 & 0,22 \\
& $\mathbf{s}$ & 1,37 & 1,33 & 0,93 & 0,83 & 0,57 \\
\multirow{3}{*}{ GII } & $\bar{x}$ & 3,75 & 4,58 & 5,25 & 5,46 & 3,97 \\
& $\mathbf{C V}$ & 0,49 & 0,72 & 0,8 & 0,95 & 0,45 \\
& $\mathbf{s}$ & 1,19 & 1,88 & 2,05 & 2,42 & 1,13 \\
\hline
\end{tabular}




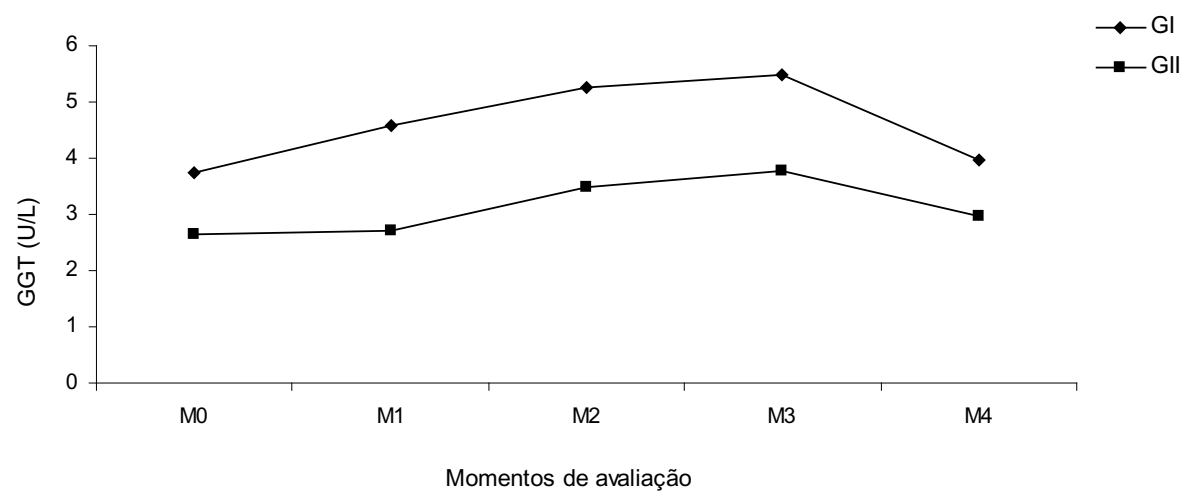

Figura 4. Variação dos valores médios de GGT (U/L) em cães anestesiados com a associação zolazepam/tiletamina em diferentes momentos.

\section{Discussão}

Há um aumento significativo na concentração sérica de enzimas hepáticas em pacientes humanos submetidos à infusão de cetamina (HU et al., 1994). Fantoni, Cortopassi e Bernardi (1999) descrevem alterações semelhantes em animais, após anestesia com cetamina, observando um aumento na concentração das enzimas hepáticas por 3-4 dias, fato não verificado quando tiopental, midazolam ou etomidato são empregados. No entanto, esses mesmos autores citam que, em pacientes humanos com disfunção hepática prévia, não houve agravamento do quadro com a utilização do referido fármaco. No presente trabalho, foi verificado comportamento semelhante com a associação zolazepam/tiletamina, em única ou mais doses. No entanto, os valores não se mantiveram fora dos valores normais ou em ascendência após 24 horas da última aplicação, apesar de ter sido verificado aumento maior na concentração de ALT, FA e GGT em GI, grupo este que recebeu 3 doses.

Mello e Cordeiro (2001) em estudo com a mesma associação em única dose para cães de diferentes faixas etárias verificaram aumento nas concentrações das enzimais hepáticas, principalmente na enzima creatinina-quinase no grupo de animais adultos e jovens. Os referidos autores alegam que os dados obtidos na pesquisa não são conclusivos para relacionar esse aumento com danos hepáticos, corroborando os achados de Greene (1996), o qual indica a associação zolazepam/tiletamina para indução anestésica em pacientes portadores de hepatopatias e discordando de Muir et al. (2001) que recomenda cautela na utilização da cetamina nesses pacientes.

A co-administração de substâncias biotransformadas no fígado pode estender a meiavida da cetamina em cães (VALADÃO, 2002). Provavelmente isso também acontece com a tiletamina que comercializada com o zolazepam, poderia provocar a maior elevação das enzimas hepáticas na espécie canina. Reves, Glass e Lubarsky (2000) relatam a biotansformação dos benzodiazepínicos por dois processos, através do sistema microssomal hepático, por conjugação glicurônica ou através da oxidação, produzindo metabólicos ativos que possuem menor potencial, porém prolongam a recuperação. Isso poderia ocorrer com a associação em questão, pois ambos os fármacos administrados podem competir pelo mesmo processo metabólico, sobrecarregando o fígado e, conseqüentemente elevando a fração das enzimas hepáticas e, com isso, retardando a biotransformação e excreção de ambos. Greene (1996) responsabiliza o zolazepam pela lenta recuperação anestésica na espécie felina, sugerindo dificuldade da biotransformação do mesmo nesta espécie. Este fato é salientado por Wong e Bandiera (1998) que administraram tiletamina, zolazepam 
ou pentobarbital, de maneira isolada, em ratos e concluíram que o zolazepam foi o responsável pelo aumento de isoenzimas do citocromo P450.

Isso justifica os dados obtidos nessa pesquisa, pois, apesar de todas as enzimas em questão se manterem dentro dos valores fisiológicos propostos por Topal et al. (2003), os animais de GI, que receberam três doses de zolazepam/tiletamina, apresentaram maiores índices de ALT, FA e GGT em todos os momentos comparados a GII, indicando o provável efeito deletério de diversas doses ao fígado.

Os valores de AST obtidos no presente estudo divergem daqueles relatados por McEwen et al. (2000), que ao avaliarem a função hepática de ovinos anestesiados com cetamina e xilazina em única dose e mantidos com halotano, observaram que tal enzima apresentou incremento gradativo até 24 horas após a administração do agente dissociativo. $\mathrm{O}$ incremento súbito observado seguida de redução podem ser justificados pela aplicação intramuscular, uma vez que a AST também é uma enzima muscular, a sua liberação pode ter ocorrido devido a tal aplicação (TENNANT, 1997).

González et al. (2002) ao utilizarem a cetamina associada a xilazina ou diazepam em coelhos em apenas uma dose, observaram que apesar das duas associações elevarem as enzimas hepáticas, as mesmas se mantiveram dentro dos valores normais. Em outro experimento, Rofael (2004) submeteu ratos a uma dose de $100 \mathrm{mg} / \mathrm{kg}$ de cetamina por via oral durante três dias consecutivos e não verificou alterações hepáticas dignas de nota. Hashimoto et al. (1991) ao compararem pacientes humanos submetidos à infusão contínua de fentanil e cetamina ou enfluorano e óxido nitroso, avaliaram a função hepática 24 e 72 horas após procedimentos cirúrgicos, de duração média de 120 minutos e observaram que o protocolo baseado na cetamina foi o que causou menores alterações no fígado.

Pacientes com lesão hepática de diferentes formas possuem dificuldade ou inabilidade em biotransformar os fármacos administrados (FOSSUM, 2002). Os derivados das fenciclidinas possuem biotransformação hepática elevada, assim sendo animais portadores de hepatopatias podem apresentar dificuldade de biotransformação da tiletamina em condições de sobredosagens, visto que os resultados desta pesquisa, em pacientes hígidos, apontam valores das enzimas hepáticas superiores com três dosagens em relação a uma única dose isolada do referido fármaco, contraindicando o emprego o emprego da associação de zolazepam/tiletamina em pacientes hepatopatas e, caso sua utilização seja necessária, esta deve ser feita com cautela. A administração de uma única dose da associação induziu elevação das enzimas hepáticas, apesar dos valores terem permanecido dentro dos limites fisiológicos para a espécie e desta elevação ser menor que a promovida pela aplicação de duas doses repetidas, indicando que, em pacientes hepatopatas, a utilização da associação zolazepam/ tiletamina deve ser feita com cautela, mesmo quando apenas uma administração for necessária.

\section{Conclusões}

Diante dos resultados obtidos nesse experimento, concluiu-se que:

Uma dose de $6,6 \mathrm{mg} / \mathrm{kg}$ de zolazepam/tiletamina por via intramuscular promove valores menores das enzimas avaliadas em relação a três doses;

A associação anestésica zolazepam/tiletamina na dose de $6,6 \mathrm{mg} / \mathrm{kg}$, seguida de duas novas aplicações de 3,3 $\mathrm{mg} / \mathrm{kg}$ elevam as enzimas hepáticas avaliadas em cães, sem ultrapassar os valores fisiológicos;

\section{Referências}

DUPRAS, J.; VACHON, P.; CUVELLIEZ, S.; BLAIS, D. Anestesia of the New Zeland rabbit using the combination of tiletamine-zolazepam and ketamine-midazolam with or without xilazine. Canadian Veterinary Journal, Ottawa, v. 42, n. 6, p. 455-460, 2001. 
DUVAL NETO, G. F. Anestésicos venosos. In: MANICA, J. Anestesiologia princípios e técnicas. 3. ed. Porto Alegre: ArtMed, 2004. p. 560-620.

FANTONI, D.; CORTOPASSI, S. R. G.; BERNARDI, M. M. Anestésicos intravenosos e outros parenterais. In: SPINOSA, A. S.; GÓRNIAK, S. L.; BERNARDI, M . M. Farmacologia aplicada a medicina veterinária. 2. ed. Rio de Janeiro: Guanabara Koogan, 1999. p. 114-124.

FOSSUM, T. W. Surgery of the liver. In: Small animal surgery. 2. ed. Saint Louis: Mosby, 2002. p. 460474.

GONZÁlEZ, G. A.; ILLERA, J. C.; SILVAN, G.; LORENZO, P. L.; ILLERA, M. Changes in hepatic and renal enzyme concentrations and heart and respiratory rates in New Zeland white rabbits after anesthetic treatments. Contemporary Topics in Laboratory Animal Science, New Jersey, v. 41, n. 6, p. 30-32, 2002.

GREENE, S. A. Hepatic disease. In: THURMON, J. C.; TRANQUILLI, W. J.; BENSON, G. J. Lumb \& Jones' veterinary anesthesia. 3. ed. Baltimore: Williams \& Wilkins, 1996. p. 791-797.

HASHIMOTO, Y.; ARAKI, I.; KUSHIKATA, T.; MURAKAWA, T.; ISHIHARA, H.; MATSUKI, A. Clinical study on total intravenous anesthesia with droperidol, fentanyl and ketamine. Effects on postoperative liver function. Massui, Tóquio, v. 40, n. 5, p. 749-753, 1991.

HEAVNER, J. E. Drug interactions. In: THURMON, J. C.; TRANQUILLI, W. J.; BENSON, G. J. Lumb \& Jones' Veterinary Anesthesia. 3. ed. Baltimore: Williams \& Wilkins, 1996. p. 35-39.

HU, J. W.; SUN, W. Z.; SUSETIO, L.; HOU, W. Y.; CHANG, Y.; LIN, S. Y.; LIU, C. C. Precipitation profile of the intravenous induction agents. Acta Anaesthesioligica Sínica, New Jersey, v. 32, n. 2, p. 105-107, 1994.

MASSONE, F. Anestesiologia veterinária, farmacologia e técnicas. 4. ed. Rio de Janeiro: Guanabara, 2003.

McEWEN, M. M.; GLEED, R. D.; LUDDERS, J. W.; STOKOL, T.; DEL PIERO, F.; ERB, H. N. Hepatic effects of halothane and isoflurane anesthesia in goats. Journal of American Veterinary Medicine Association, Schaumburg, v. 217, n. 11, p. 1697-1700, 2000.

MELLO, J. R. B.; CORDEIRO, M. R. O. Avaliação da associação tiletamina-zolazepam de três diferentes faixas etárias. Arquivos da Faculdade de Veterinária - UFRS, Porto Alegre, v. 29, n. 1, p. 14-24, 2001,

MEYER, D. J.; COLES, E. H.; RICH, L. J. Medicina de laboratório veterinária: interpretação e diagnóstico. São Paulo: Roca, 1995.

MUIR, W. W.; HUBBEL, J. A. E.; SKARDA, R. T. BEDNARSKI, R. M. Manual de anestesia veterinária. 3. ed. Porto Alegre: Artmed, 2001.

REVES, J. G.; GLASS, P. S. A.; LUBARSKY, D. A. Nonbarbiturate intravenous anesthetics. In: MILLER, R. D. Anesthesia. 15. ed. Philadelphia: Churchil Livingstone, 2000. v. 2, p. 546-590.

ROFAEL, H. Z. Effect of ketamine pretreatment on cocaine-mediated hepatotoxicity in rats. Toxicology Letters, New Jersey, v. 152, n. 3, p. 213-222, 2004.

SANTOS, G. J. G.; MASSONE, F.; MATTOS JUNIOR, E.; TRINCA, L. A. Avaliação bispectral das associações anestésicas: atropina-cetaminaxilazina, levomepromazina-tileramina-zolazepam e levomepromazina-cetamina-midazolam em cães. Revista Científica Medicina Veterinária Pequenos Animais Animais Estimação, Curitiba, v. 2, n. 6, p. 109-113, 2004.

SAVVAS, I.; PLEVRAKI, K.; RAPTOPOULOS, D.; KOUTINAS, A. F. Blood gas and acid-base status during tiletamine/zolazepam anaesthesia in dogs. Veterinary Anaesthesia and Analgesia, New York, v. 32, n. 2, p. 94-100, 2005.

TENNANT, B. C. Hepatic Function. In: KANEKO, J. J.; HARVEY, J. W.; BRUSS, M. L. Clinical biochemistry of domestic animals. 15. ed. Academic Press: San Diego, 1997. p. 240-263.

TOPAL, A.; GÜL, N.; ILÇÖL, Y.; GÖRGÜL, O. S. Hepatic effects of halothane, isoflurane or sevoflurane anaesthesia in dogs. Journal of Veterinary Medicine Association, Schaumburg, v. 50, n. 10, p. 530-533, 2003.

VALADÃO, C. A. A. Anestésicos dissociativos. In: FANTONI, D. T.; CORTOPASSI, S. R. G. Anestesia em cães e gatos. São Paulo: Roca, 2002. p. 165-173.

WONG, A.; BANDIERA, S. M. Induction of hepatic cytocrome $\mathrm{P} 4502 \mathrm{~B}$ and $\mathrm{P} 4503 \mathrm{~A}$ isoenzymes in rats by zolazepam, a constituent of Telazol. Biochemistry Pharmacology, New York, v. 55, n. 2, p. 201-207, 1998. 